\title{
Manifestações retinianas em pacientes portadores de anemia de células falciformes
}

\author{
Retinal sings in patients with sickle cell disease
}

Edmundo Frota de Almeida Sobrinho', João Carlos de Pina Saraiva², James Nunes da Silva ${ }^{3}$, Ana Paula Siqueira da Silva ${ }^{4}$, Rafaela Cavalcante de Lima $^{4}$

\section{ResUmo}

A Organização Mundial de Saúde estima que mais de 5\% da população mundial seja portadora de algum tipo de hemoglobinopatia. Dentre essas encontramos a anemia de células falciformes, que tem seu principal efeito lesivo sobre a vasculatura periférica. Na retina, as lesões falciformes possuem fisiopatologia e classificação bem definidas. O objetivo é identificar as manifestações retinianas à anemia falciforme em pacientes encaminhados ao Hospital Bettina Ferro de Souza a partir do Hemocentro do Estado do Pará - HEMOPA. Métodos: No Serviço de Oftalmologia do Hospital Universitário Bettina Ferro de Souza realizou-se em cinquenta pacientes portadores de anemia de células falciformes, sendo 37 genótipo SS e 13 genótipo SC, foram acompanhados pelo ambulatório de anemia falciforme do HEMOPA e selecionados aleatoriamente, sendo submetidos à entrevista para registro de sexo; idade; cor; genótipo; alterações oculares; medicamentos utilizados. exame oftalmológico, incluindo angiofluoresceínografia nos casos com alteração retiniana. Resultados: Registro em protocolo de pesquisa e posteriormente submetidos à análise estatística utilizando o teste estatístico Qui-quadrado e p $<0,05$. Oitenta e oito por cento dos pacientes estudados não possuíam qualquer lesão retiniana falciforme, $3 \%$ apresentaram oclusão vascular periférica, em $2 \%$ evidenciou-se placa pigmentada, e $7 \%$ apresentaram lesões não compatíveis com a doença falciforme; quanto ao sexo houve proporcionalidade de $50 \%$ para ambos; faixa etária de maior predominância foi a de $11 \mathrm{e}$ 15 anos com 38\%,74\% enquandraram-se no genótipo SS e 26\% no SC. Em relação ao uso de medicamentos, notou-se maior prevalência de alterações oculares nos pacientes que faziam uso do ácido fólico isolado com 5\%, em contraste com aqueles em uso da associação hidróxiuréia e ácido fólico em que todos $(27 \%)$ possuíam exame fundoscópico normal. Todos os pacientes $(29 \%)$ com hemoglobina fetal acima de $10 \%$ possuíam retina sem alterações. Conclusão: Poucos casos de lesões retinianas foram observados no grupo estudado, ainda assim esta pesquisa reafirma a importância da realização do exame oftalmológico de maneira precoce e periódica, visto que, a retinopatia falciforme é fato bem documentado e suas complicações podem resultar em amaurose.

Descritores: Anemia falciforme; Retinopatias; Hemoglobinopatias; Hidroxiuréia

\footnotetext{
${ }^{1}$ Doutor, Professor Adjunto de Oftalmologia da Universidade Federal do Pará - UFPA - Belém (PA), Brasil; Professor Adjunto da Universidade do Estado do Pará - UEPA - Belém (PA)- Brasil; Preceptor da Residência Médica em Oftalmologia do Hospital Bettina Ferro de Souza - UFPA - Belém (PA), Brasil.

${ }^{2}$ Médico Hematologista da Fundação Centro de Hemoterapia e Hematologia do Pará - HEMOPA - Belém (PA), Brasil;

${ }^{3}$ Residente de Oftalmologia do Hospital Universitário Bettina Ferro de Souza, Belém (PA), Brasil;

${ }^{4}$ Acadêmica do $6^{\circ}$ ano do Curso de Medicina da Universidade do Estado do Pará - UEPA - Belém (PA), Brasil.

Trabalho realizado no Hospital Universitário Bettina Ferro de Souza, Belém (PA), Brasil

Os autores declaram inexistir conflitos de interesse

Recebido para publicação em: 14/12/2009 - Aceito para publicação em 7/8/2011
}

Rev Bras Oftalmol. 2011; 70 (5): 284-9 


\begin{abstract}
The World Health Organization counts that more than $5 \%$ of the world's population carry some type of hemoglobinopathy. Among them we find the sickle cell aneamia that presents its main harmful effect on the peripheral vasculature. In the retina, the falciform lesions have a well defined physiopathology and classification. To identify the retinal manifestations caused by the falciform aneamia in patients directed to the Bettina Ferro de Souza Hospital from the Hemocenter of the State of Pará - HEMOPA, in the months of September through December of 2006. Methods: Ophthalmologic examination was performed in the Department of Ophthalmology of the Bettina Ferro de Souza Hospital. and fifty patients with sickle cell aneamia (SS or SC) followed by the department of falciform aneamia of the Hematologia e Hemoterapia do Pará - HEMOPA have been randomly selected and submitted to interview in order to register their: sex; age; color; genotype; ocular alterations; medicines taken. Ophthalmologic examination was performed in the Department of Ophthalmology of the Bettina Ferro de Souza Hospital. It consisted of: evaluation of the acuity of vision with and without correction, indirect biomicroscopy, indirect binocular ophthalmoscopy, and, in case the latter presented alterations, a complementary study with angiofluoresceinography would be carried through. Results: The outcomes have been registered in research protocol and later submitted to the statistic analysis using the Qui-square test, adopting, as level of significance, $\mathrm{p}<0,05$. Eighty-eight percent of the patients did not present any falciform retinal sign, $3 \%$ presented peripheral vascular occlusion; in $2 \%$, pigmented plate was shown, and $7 \%$ presented injuries which were not compatible with the falciform disease; there was a proportionality of $50 \%$ for males and females; there was a higher predominance (38\%) of people aged from 11 and $15 ; 74 \%$ had SS genotype and 26\% SC genotype; in relation to the use of medicines there was a higher prevalence of ocular alterations in patients who had made use of the folic acid isolated, with $5 \%$, in contrast with those who had used the hidroxyurea association and folic acid, when all (27\%) presented normal fundoscopic examination; all the patients (29\%) that showed fetal hemoglobin rate above $10 \%$ had retina without alterations. Conclusion: Few cases of retinal signs have been observed in the studied group, however this research does not diminish the importance of early and periodic ophthalmologic examination, since falciform retinopathy is largely registered and its complications can lead to amaurosis.
\end{abstract}

Keywords: Sickle cell disease; Retinopathies; Hemoglobinopathies

\section{INTRODUÇãO}

A Organização Mundial de Saúde estima que mais de $5 \%$ da população mundial seja portadora de algum tipo de hemoglobinopatia ${ }^{(1)}$. Estima-se o surgimento de 700 a 1.000 novos casos anuais de doença falciforme no Brasil e prevalência de mais de 2 milhões de portadores do gene $\mathrm{HbS}^{(2)}$.

A doença de células falciformes pode causar complicações oftalmológicas, que resultam em comprometimento da visão $0^{(3)}$. Na retina, essas alterações tornam-se mais evidentes e destrutivas, podendo ocasionar amaurose, apesar de sua evolução lenta e muitas vezes benigna $^{(4)}$.

O diagnóstico precoce através do exame oftalmológico periódico, com ênfase na oftalmoscopia da periferia retiniana é de extrema importância, pois permite a instituição de tratamento adequado em tempo hábil, evitando a progressão da doença, bem como o dano permanente à visão ${ }^{(5)}$.

O objetivo foi identificar as manifestações retinianas associadas à anemia falciforme em pacientes encaminhados ao Hospital Bettina Ferro de Souza a partir da Fundação Centro de Hemoterapia e Hematologia do Pará.

\section{Métodos}

Após aprovação do Conselho de Ética do HEMOPA, foram examinados 50 pacientes portadores de anemia de células falciformes (genótipos SS e SC), acompanhados no ambulatório de anemia falciforme do HEMOPA, nos meses de setembro a dezembro de 2006. Dessa forma, realizou-se um inquérito, caracterizandose um estudo individuado, observacional e descritivo.

O diagnóstico de anemia de células falciformes foi feito através da eletroforese da hemoglobina acetato celulose em $\mathrm{pH}$ alcalino, da dosagem de hemoglobina fetal $(\mathrm{HbF})$ realizada pelo método de desnaturação alcalina e pelo hemograma de citometria de fluxo.

Os pacientes, habitantes de uma região de alta concentração de oxigênio atmosférico, foram submetidos no HEMOPA à entrevista para registro de genótipo, uso de medicamentos e nível de hemoglobina fetal. Em seguida foram encaminhados ao Serviço de Oftalmologia do Hospital Universitário Bettina Ferro de Souza, onde foram realizados os seguintes exames:

- avaliação da acuidade visual com e sem correção;

- biomicroscopia do segmento anterior.

Todos os casos que apresentaram alterações 
vasculares compatíveis com a doença de base em questão foram submetidos à angiofluoresceínografia, sendo os resultados anexados ao prontuário dos respectivos pacientes e arquivados na Fundação Hemopa, além de registrados em protocolo de pesquisa, e posteriormente submetidos à análise estatística pelo teste Qui-quadrado com $\mathrm{p}<0,05$.

\section{Resultados}

Foram analisados 50 pacientes portadores de anemia de células falciformes sendo distribuídos em relação ao sexo ( 25 masculino e 25 feminino) num total de 100 olhos.

A faixa etária mais prevalente foi a de 11 a 15 $\operatorname{anos}(n=19,38 \%)$ e a cor predominante foi a parda (98\%).
O genótipo mais frequente foi o SS presente em $74 \%(\mathrm{n}=37)$ dos pacientes $(\mathrm{p}<0,05)$.

Oitenta e oito por cento $(\mathrm{n}=88)$ dos pacientes portadores de anemia falciforme não apresentavam alterações retinianas (Tabela 1).

Dos usuários do quimioterápico hidroxiuréia, 27\% ( $n=27$ ) possuíam exame fundoscópico normal, e aqueles que desenvolveram alteração $(n=3,3 \%)$, essas não eram decorrentes de doença falciforme (Tabela 2).

Observou-se que todas as alterações retinianas encontradas pertenciam aos pacientes de genótipo SS, diferentemente daqueles de genótipo SC, que não apresentaram nenhuma alteração ocular (Tabela 3).

Oss pacientes que apresentaram $\mathrm{HbF}$ maior ou igual a $10 \%$, 29\% $(n=29)$ não possuíam alteração vascular retiniana decorrente da anemia de células falciformes (Tabela 4).

Tabela 1

\begin{abstract}
Alterações da retina dos pacientes portadores de anemia de células falciformes atendidos no ambulatório da Fundação de Hematologia e Hemoterapia do Estado do Pará, Belém - PA, setembro a dezembro de 2006
\end{abstract}

\begin{tabular}{lcc}
\hline Alterações da retina & $\mathbf{n = 1 0 0}$ & frequência (\%) \\
\hline Normal(*) & 88 & 88,0 \\
Oclusão vascular periférica & 03 & 3,0 \\
Placa pigmentada & 02 & 2,0 \\
Outros & 07 & 7,0 \\
Total & 100 & 100,0 \\
\hline
\end{tabular}

Fonte: Protocolos de pesquisa (*) $\mathrm{p}<0,05$ (Qui-quadrado)

Tabela 2

Relação entre uso de medicamentos e alteração retiniana nos pacientes portadores de anemia de células falciformes atendidos no ambulatório da Fundação de Hematologia e Hemoterapia do Estado do Pará, Belém - PA, setembro a dezembro de 2006

\begin{tabular}{|c|c|c|c|c|c|c|c|c|}
\hline \multirow[b]{3}{*}{ Alteração da Retina } & \multicolumn{6}{|c|}{ Medicamentos } & & \\
\hline & \multicolumn{2}{|c|}{ Sem uso } & \multicolumn{2}{|c|}{$\begin{array}{c}\text { Hidroxiuréia + } \\
\text { Ácido Fólico }\end{array}$} & \multicolumn{2}{|c|}{ Ácido Fólico } & \multicolumn{2}{|c|}{ Total } \\
\hline & $\mathbf{N}$ & $\%$ & $\mathbf{N}$ & $\%$ & $\mathbf{N}$ & $\%$ & $\mathbf{N}$ & $\%$ \\
\hline Normal & 4 & 4,0 & 27 & 27,0 & 57 & 57,0 & 88 & 88,0 \\
\hline Oclusão Vascular Periférica & 0 & 0,0 & 0 & 0,0 & 3 & 3,0 & 3 & 3,0 \\
\hline Placa Pigmentada & 0 & 0,0 & 0 & 0,0 & 2 & 2,0 & 2 & 2,0 \\
\hline Outros & 0 & 0,0 & 3 & 3,0 & 4 & 4,0 & 7 & 7,0 \\
\hline Total & 4 & 4,0 & 30 & 30,0 & 66 & 66,0 & 10 & 100,0 \\
\hline
\end{tabular}


Tabela 3

Relação entre genótipo e alteração retiniana dos pacientes portadores de anemia de células falciformes atendidos no ambulatório da Fundação de Hematologia e Hemoterapia do Estado do Pará, Belém - PA

\begin{tabular}{lccccccc}
\hline & \multicolumn{3}{c}{ Genótipos } & \multicolumn{2}{c}{ SS } & \multicolumn{2}{c}{ Total } \\
\cline { 2 - 5 } Alteração da retina & $\mathbf{N}$ & SC & N & \% & N & $\%$ \\
\hline Normal & 26 & 26,0 & 62 & 62,0 & 88 & 88,0 \\
Oclusão vascular periférica & 0 & 0,0 & 3 & 3,0 & 3 & 3,0 \\
Placa pigmentada & 0 & 0,0 & 2 & 2,0 & 2 & 2,0 \\
Outros & 0 & 0,0 & 7 & 7,0 & 7 & 7,0 \\
TOTAL & $\mathbf{2 6}$ & $\mathbf{2 6 , 0}$ & $\mathbf{7 4}$ & $\mathbf{7 4 , 0}$ & $\mathbf{1 0 0}$ & $\mathbf{1 0 0 , 0}$ \\
\hline
\end{tabular}

Fonte: Protocolos de pesquisa

Tabela 4

\begin{abstract}
Relação entre hemoglobina fetal e alteração retiniana dos pacientes portadores de anemia de células falciformes atendidos no ambulatório da Fundação de Hematologia e Hemoterapia do Estado do Pará, Belém - PA, setembro a dezembro de 2006
\end{abstract}

\begin{tabular}{|c|c|c|c|c|c|c|c|c|}
\hline \multirow[b]{3}{*}{ Alteração da retina } & \multicolumn{6}{|c|}{ Hemoglobina Fetal } & & \\
\hline & \multicolumn{2}{|c|}{0 a 4} & \multicolumn{2}{|c|}{5 a 9} & \multicolumn{2}{|c|}{$\geq \mathbf{1 0}$} & \multicolumn{2}{|c|}{ Total } \\
\hline & $\mathbf{N}$ & $\%$ & $\mathbf{N}$ & $\%$ & $\mathbf{N}$ & $\%$ & $\mathbf{N}$ & $\%$ \\
\hline Normal & 13 & 13,0 & 46 & 46,0 & 29 & 29,0 & 88 & 88,0 \\
\hline Oclusão vascular periférica & 1 & 1,00 & 2 & 2,0 & 0 & 0,0 & 3 & 3,0 \\
\hline Placa pigmentada & 0 & 0,00 & 2 & 2,0 & 0 & 0,0 & 2 & 2,0 \\
\hline Outros & 2 & 2,00 & 2 & 2,0 & 3 & 3,0 & 7 & 7,0 \\
\hline TOTAL & 16 & 16,0 & 52 & $\mathbf{5 2 , 0}$ & 32 & 32,0 & 10 & 100,0 \\
\hline
\end{tabular}

Fonte: Protocolos de pesquisa

Sintomas oculares estavam presentes em $54 \%$ $(\mathrm{n}=27)$ dos pacientes, destacando-se a dificuldade visual $(\mathrm{n}=12,44,44 \%)$. O genótipo mais frequente foi o SS, presente em $74 \%$ dos pacientes $(\mathrm{p}<0,05)$.

\section{Discussão}

As hemoglobinopatias afetam a população brasileira com frequências variáveis, refletindo a sua característica de formação étnica ${ }^{(6)}$.

Neste estudo, os 50 pacientes examinados tiveram uma distribuição igualitária em ambos os sexos, diferente dos demais outros nos quais houve predominância do sexo feminino ${ }^{(6)}$.

A idade dos pacientes examinados teve como limite inferior o valor de 18 meses, e como limite superior 36 anos, média entre 11 e 15 anos com $38 \%(n=19)$ dos casos, o que corrobora com a literatura.

Gonçalves et al. ${ }^{(3)}$, Garcia et al. ${ }^{(7)}$ e Talbot et al. ${ }^{(8)}$ concordam que a faixa etária de maior ocorrência das lesões retinianas proliferativas da doença falciforme predominam a partir dos 20 anos de idade, entretanto neste trabalho não houve esta alteração, e a maioria dos pacientes tinha exame normal.

Goldberg $^{(9)}$ estabeleceu uma descrição minuciosa a respeito da fisiopatologia e classificação da retinopatia da anemia de células falciformes. As alterações foram divididas em proliferativas e não proliferativas baseadas na presença ou ausência de anastomoses arteriovenosas. Os estágios préproliferativos (I e II de Goldberg) acontecem em dois terços dos pacientes, independente do genótipo ${ }^{(10)}$.

Dentre as alterações encontradas no atual estudo, nenhuma se enquadrava no padrão proliferativo da ane- 
mia de células falciformes. Este fato foi ratificado pelo estudo de Talbot et al. (1992) ${ }^{(8)}$, o qual relacionou o não aparecimento deste tipo de lesão com variantes hematológicas, como por exemplo, nível de $\mathrm{HbF}$ acima de $10 \%$.

A ausência de retinopatia proliferativa neste estudo também pode ser justificada pelo uso do quimioterápico Hidroxiuréia em alguns pacientes, a qual, segundo Epstein ${ }^{(10)}$, leva a um aumento da hemoglobina fetal, sendo que $27 \%(n=27)$ dos pacientes usuários desta droga não desenvolveram alteração retiniana, e aqueles que desenvolveram $(n=03,03 \%)$ não eram decorrentes doença falciforme.

Gonçalves et al. ${ }^{(3)}$, Bonanomi et al. ${ }^{(4)}$ e Garcia et al. ${ }^{(7)}$ fizeram alusão em seus estudos ao modo de instalação da lesão vascular proliferativa, uma vez que quanto mais insidiosa, maior a chance de desenvolver esse tipo de lesão, observada portanto em idades mais elevadas, principalmente acima de 20 anos. Clarkson ${ }^{(11)}$, em seu estudo, ainda relatou que estas lesões estão mais relacionadas com o genótipo SC.

O estudo presente pressupõe a veracidade dos resultados encontrados pelos demais autores, uma vez que apenas $6 \%(n=12)$ dos pacientes possuíam idade acima de 20 anos e $26 \%$ ( $n=13)$ genótipo SC, o que pode ser explicado pela pouca expressividade destes fatores dentro da totalidade da amostra.

As alterações oculares da anemia de células falciformes são na maioria das vezes assintomáticas, quando sintomáticas aparecem em fases mais tardias da doença ocular ${ }^{(2)}$. Isto foi constatado no atual estudo, pois em $83.33 \%(n=45)$ dos pacientes que possuíam sintomas visuais apresentavam exame oftalmológico normal.

Segundo Gonçalves et al. ${ }^{(3)}$, a maioria dos pacientes com hemoglobinopatia SC possui um curso clínico discreto com exames hematológicos próximos da normalidade e episódios infrequentes de fenômenos vasooclusivos. Os pacientes homozigóticos (SS) para anemia de células falciformes possuem fenômenos vaso-oclusivos frequentes e um curso clínico mais severo, porém a retinopatia proliferativa falciforme é significativamente menos frequente.

De acordo com Fox et al. ${ }^{(12)}$, a retinopatia falciforme ocorre predominantemente nos pacientes de genótipo SC, pois apresentam fenômenos vaso-oclusivos de intensidade moderada, ou seja, capaz de desenvolver oclusões na vasculatura retiniana periférica, estimulando o desenvolvimento da doença proliferativa da retina.

O presente estudo não constatou tais afirmações, visto que todas as alterações retinianas encontradas pertenciam a pacientes de genótipo SS, diferentemente da- queles de genótipo SC, que não possuíram nenhuma alteração ocular. Tal fato ocorreu porque as lesões que predominam no genótipo SC são do tipo proliferativa (Fox et al. ${ }^{(12)}$ ), as quais não foram encontradas nos pacientes do estudo.

O estudo em questão concordou com a relação de positividade entre o valor da $\mathrm{HbF}$ maior ou igual a $10 \%$ e a diminuição da ocorrência da oclusão vascular periférica, já que nenhum dos pacientes com HbF maior ou igual ao valor referido, possuía alteração vascular retiniana decorrente da anemia de células falciformes. Segundo Silveira et al. ${ }^{(13)}$, a relação entre $\mathrm{HbF}$ e a gravidade das manifestações clínicas da doença não é tão simples, tendo em vista que alguns pacientes com concentração de $\mathrm{HbF}$ baixa $(<10 \%)$ desenvolvem um curso brando da doença, em contraste com outros que, apesar possuírem elevados níveis de $\mathrm{HbF}$, desenvolvem crises álgicas frequentes. Epstein ${ }^{(10)}$, relatou, em seu artigo de revisão, que a Hidróxiuréia (HU) é uma droga capaz de estimular a produção de HbF. Isto ocorre através da sua ação direta sobre os precursores eritróides, reprogramando-os para uma maior produção desse tipo de hemoglobina ${ }^{(14)}$. O ácido fólico, por sua vez, segundo Teixeira et al. ${ }^{(15)}$ tem como objetivo tornar-se substrato para a produção da série vermelha, a qual se encontra aumentada em decorrência da anemia hemolítica, não tendo, portanto, sua ação, interferência quanto à ocorrência de oclusão vascular periférica.

\section{Conclusão}

A pesquisa conclui que dentre os pacientes estudados portadores de anemia de células falciformes do ambulatório da Fundação de Hematologia e Hemoterapia do Estado do Pará , no período de setembro a dezembro de 2006, 5\% ( $\mathrm{n}=05)$ apresentaram retinopatia falciforme não proliferativa, dentre os quais $3 \%(n=03)$ dos casos referiram-se à oclusão vascular periférica e $2 \%(n=02)$ à placa retiniana pigmentada, não tendo sido encontrado nenhum caso de retinopatia proliferativa falciforme.

Embora não tenha sido observado nenhum caso de retinopatia proliferativa resultante da anemia de células falciformes, este estudo não diminui a importância da realização do exame oftalmológico de maneira precoce e periódica, visto que as lesões não proliferativas podem ser precursoras de retinopatia proliferativa que podem, de maneira desastrosa, resultar em amaurose. 


\section{REFERÊNCIAS}

1 - Bonini-Domingos AC, Viana-Baracioli LMS, Bonini-Domingos CR. Identificação de variantes de hemoglobina em doadores de sangue. Rev Bras Hematol Hemoter. 2004;26(1):57-9.

2 - Brasil. Ministério da Saúde. Agência Nacional de Vigilância Sanitária. Manual de diagnóstico e tratamento de doenças falciformes. Brasília: ANVISA; 2001. p. 97-104.

3 - Gonçalves JCM, Braga JAP, Nione AS, Simoceli RA, Yamamoto M. Retinopatia falciforme em crianças. Arq Bras Oftalmol. 1990;53(4):158-61.

4 - Bonanomi MT, Cunha SL, de Araújo JT. Funduscopic alterations in SS and SC hemoglobinopathies. Study of a Brazilian population. Ophthalmologica. 1988;197(1):26-33.

5 - Herrick JB. Peculiar elongated and sickle-shaped red blood corpuscles in a case of severe anemia. 1910. Yale J Biol Med., 2001;74(3):179-84.

6 - Carney, M.D;; JAMPOL, L.M. Epiretinal membranes in sikle cell retinopathy. Arch. Ophthalmol. 1987;105:214-17.

7 - Garcia CAA, Fernandes MZ, Uchoa UBC, Cavalcante BM, Uchoa RAC. Achados fundoscópicos em crianças portadoras de anemia falciforme no estado do Rio Grande do Norte. Arq Bras Oftalmol. 2002;65(6):615-8.

8 - Talbot JF, Bird AC, Maude GH, Acheson RW, Moriarty BJ, Serjeant GR. Sickle cell retinopathy in Jamaican children: further observation from a cohort study. Br J Ophthalmol. 1988;72(10):727-32.
9 - Goldberg MF. Classification and pathogenesis of proliferative sickle retinopathy. Am J Ophthalmol. 1971;71(3):649-65.

10 - Epstein FH. Pathogenesis and treatment of sickle cell disease. N Engl J Med. 1997;337(11): 762-9. Review.

11 - Clarkson JG. The ocular manifestations of sickle-cell disease: a prevalence and natural history study. Trans Am Ophthalmol Soc. 1992;90:481-504.

12 - Fox PD, Higgs DR, Serjeant GR. Influence of alpha thalassaemia on the retinopathy of homozygous sickle cell disease. Br J Ophthalmol. 1993;77(2):89-90.

13 - Silveira MM, Fonseca LM. A complexa fisiopatologia dos episódios vaso-oclusivos na anemia falciforme. Rev Cienc Farm. 2002;23(1):25-46.

14 - Khayat AS, Guimarães AC, Cardoso PC, Lima PD, Bahia MO, Antunes LMG, Burbano RR. Mutagenicity of hidroxyurea in lymphocytes from patients with sickle cell disease. Genet Mol Biol. 2004;27(1):115-7.

15 - Teixeira SM, Cortellazzi LC, Grotto HZ. Effect of hidroxyurea on $\mathrm{G}$ gamma chain fetal hemoglobin synthesis by sickle-cell disease patients. Braz J Med Biol Res. 2003;36(10):1289-92.

\section{Endereço para correspondência: \\ Edmundo Frota de Almeida Sobrinho \\ Av. Conselheiro Furtado, $\mathbf{n}^{\circ} 2818$-apto 2818 \\ CEP 60063060 - Belém-PA \\ Telefone: 91-32618102}

E-mail: ealmeida@amazon.com.br 